\title{
Assemblies of magnetite nanoparticles extracted from magnetotactic bacteria: A magnetic study
}

\author{
A. M. Huízar-Félix, ${ }^{1,2}$ D. Muñoz, ${ }^{3}$ I. Orue, ${ }^{4}$ C. Magén, ${ }^{5,6,7}$ A. Ibarra, ${ }^{5}$ J. M. Barandiarán, ${ }^{1,2}$ \\ A. Muela, ${ }^{2,3}$ and M. L. Fdez-Gubieda ${ }^{1,2}$ \\ ${ }^{1}$ Departamento de Electricidad y Electrónica, Universidad del País Vasco (UPVIEHU), Leioa 48940, Spain \\ ${ }^{2}$ BCMaterials, Technological Park of Biscay, Derio 48160, Spain \\ ${ }^{3}$ Departamento de Inmunología, Microbiología y Parasitología, Universidad del País Vasco (UPV/EHU), \\ Leioa 48940, Spain \\ ${ }^{4}$ SGIker Medidas Magnéticas, Universidad del País Vasco (UPVIEHU), Leioa 48940, Spain \\ ${ }^{5}$ Laboratorio de Microscopías Avanzadas, Universidad de Zaragoza, Instituto de Nanociencias de Aragón, \\ Zaragoza 50018, Spain \\ ${ }^{6}$ Departamento de Física de la Materia Condensada, Universidad de Zaragoza, Zaragoza 50009, Spain \\ ${ }^{7}$ Fundación ARAID, Zaragoza 50018, Spain
}

(Received 30 September 2015; accepted 1 February 2016; published online 10 February 2016)

\begin{abstract}
Self-assembly has emerged as a suitable technique for tuning the properties of nanoparticles. In this work, we report the self-assembly of magnetosomes assisted by an external magnetic field. The magnetosomes are magnetite nanoparticles biomineralized by magnetotactic bacteria Magnetospirillum gryphiswaldense. These nanoparticles present truncated cubo-octahedral morphology with a mean diameter of $\approx 36 \mathrm{~nm}$ and are surrounded by a lipid bilayer membrane with a thickness $\approx 2-4 \mathrm{~nm}$. The use of the appropriate preparation conditions, such as initial colloidal concentration and magnetic fields applied during deposition allowed us to obtain very reproducible self-assembled 2D patterns. Homogeneous ensembles of magnetosomes onto silicon and carbon surfaces are composed of elongated structures in the form of wide chains that cover a large area of the substrates. Transmission electron microscopy image and off-axis electron holography showed the map of the stray magnetic fields produced by these assemblies. The induced magnetic anisotropy was analyzed by measuring the hysteresis loops of the assemblies at different angles in a magneto-optical Kerr effect magnetometer. The evolution of the coercive field and remanence verified the presence of well-defined patterns. The experimental results were analyzed on the based of a biaxial model. C 2016 AIP Publishing LLC.

[http://dx.doi.org/10.1063/1.4941835]
\end{abstract}

The regular arrangement of nanoscale materials opens up diverse opportunities for developing miniaturized electronic, optoelectronic, information storage materials, and biomedical applications like biosensors and hyperthermia. ${ }^{1-7}$ A simple method to fabricate these ordered structures is the selfassembly, which consists in the autonomous organization of components into patterns and structures due to the balance between electrostatic forces, surface tension, entropy, and substrate affinity. ${ }^{8}$ The precise organization of the nanoparticles in these superstructures is strongly dependent on their size, size distribution, shape, and concentration. In magnetic materials, it is also affected by the exchange coupling phenomena, the magnetic anisotropy, and the dipolar interactions. The magnetic behavior of the resulting close-packed structures is determined by the dipolar magnetic interactions. ${ }^{9-11}$ Most selfassembly studies are focused on coated nanoparticles, ${ }^{2}$ where agglomeration effects can be mostly avoided and the formation of ordered structures ${ }^{10}$ is possible. Magnetite nanoparticles synthesized by magnetotactic bacteria, usually called magnetosomes, are good self-assembled promoters ${ }^{12}$ because of their extremely low dispersity of sizes, shapes, and reproducibility. Magnetosomes present very high structural, chemical, and magnetic quality due to the genetic control that the bacteria exert during the biomineralization process. ${ }^{13}$ In particular, we work with the species Magnetospirillum gryphiswaldense that synthesizes magnetite with cubo-octahedral shape and mean diameter $\approx 40 \mathrm{~nm} .{ }^{14}$ More interestingly, these nanoparticles are covered by lipid bilayer membrane with a thickness of $\approx 2-4 \mathrm{~nm}$ (Ref. 14) that favors electrostatic stability avoiding aggregation and help controlling the minimum distances between the nanoparticles during the self-assembly. Besides, the lipid bilayer also confers a high biocompatibility making the magnetosomes promising candidates for applications in biomedicine like targeted drug delivery, magnetic resonance imaging (MRI), biosensors, and hyperthermia for cancer treatment. ${ }^{15-17}$ Even though several works have already performed the magnetic study of extracted-magnetosomes chains, ${ }^{12,18-26}$ most of them refers to a single chain or inside the bacteria. ${ }^{24-26}$ In fact, few works have been devoted to obtain wide chains of magnetosomes ${ }^{12}$ by magnetically assisted assembling. The self-assembly effects provide a tool to finely tune the magnetic properties. This is particularly true for the effective magnetic anisotropy, which in highly organized arrangements is strongly affected by dipolar inter-particle interactions. ${ }^{12}$ Herein below, we will focus on this question by analyzing the magnetic properties of magnetosomes when they are self-organized in chainshaped macrostructures as a result of the application of an external magnetic field. For this purpose, we combine powerful structural techniques as the Transmission Electron Microscopy (TEM) with magnetic characterization by Off-axis Electron Holography and Magneto-Optical Kerr Effect (MOKE) hysteresis loop measurements. Electron holography allows mapping of the magnetic stray field lines and therefore provides a picture of the magnetic ground state at remanence. Our aim is to 
correlate the self-assembly structural properties with the magnetization response to external magnetic fields. Because of its intrinsic local nature, MOKE arises as a suitable option for magnetic anisotropy characterization. Measurements as a function of the angle between chains and the external magnetic field can be quickly and carefully performed. Note that these kinds of 2D structures are usually below the detection threshold of bulk magnetometric techniques. Finally, the experimental results will be discussed on the basis of a biaxial model.

Magnetosomes were extracted from the bacterium Magnetospirillum gryphiswaldense strain MSR-1 (DMSZ 6631). As in a previous work, ${ }^{27} M$. gryphiswaldense was cultured in a flask standard medium (FSM) described by Heyen and Schüler ${ }^{28,29}$ with $\mathrm{Fe}(\mathrm{III})$-citrate added at a final concentration of $100 \mu \mathrm{M}$ (see supplementary material ${ }^{30}$ ). Magnetosomes were isolated according to the protocol described by Grünberg et al., ${ }^{31}$ with minor modifications. Briefly, cultured magnetotactic cells were collected by centrifugation, suspended in 20 mM HEPES-4 mM EDTA (pH 7.4), and disrupted by two passes through a French Pressure Cell Press FA-078 at 20000 psi. Lysates were centrifuged at $600 \times \mathrm{g}$ for $5 \mathrm{~min}$ to remove cell debris. Then, the magnetosomes were collected from the supernatant using a magnetic rack, rinsed with $10 \mathrm{mM}$ HEPES$200 \mathrm{mM} \mathrm{NaCl}(\mathrm{pH} \mathrm{7.4)}$, and finally suspended in deionized water ( $\mathrm{pH}$ 7.4). In order to avoid the presence of clumps, the isolated magnetosomes were sonicated in deionized water under sonication at $50 \mathrm{~W}$ during 90 cycles ( $15 \mathrm{~s}$ on and $5 \mathrm{~s}$ off) with an Ultrasonic Processor VCX130. The sample was placed on ice during the sonication to avoid the sample overheating. Subsequently, the magnetosomes were separated from the cellfree extract by three passes with deionized water through a homemade washing magnetic column incorporated to an electromagnet, to remove any cell debris. Finally, the so-collected magnetosomes were redispersed in deionized water. The purified magnetosomes were deposited by the drop coating method $^{15}$ under a magnetic field, onto silicon substrates previously cleaned with ethanol. The most common technique to form assembled nanoparticles uses solvent evaporation to increase the resulting particle concentration while keeping a high degree of ordering. In this study, water was used as solvent, in order to avoid damage on the lipid membrane, and was quickly evaporated using infrared lamp during the deposition. These conditions favor the particle concentration just below the liquid surface, wherein surface tension and lipid membrane act as stabilizers of the assembly. In particular, $5 \mu \mathrm{l}$ of the aqueous dispersion of magnetosomes $(35 \mu \mathrm{g} / \mathrm{ml})$ was deposited onto a silicon substrate located between the poles of an electromagnet. A magnetic field of $0.4 \mathrm{~T}$ was applied while the drop was dried under an infrared lamp to reduce the drying time $(\approx 5 \mathrm{~min})$. These deposition conditions were carefully assigned in order to optimize the self-assembly structure (see supplementary material $^{30}$ ). Measurements of Scanning Electron Microscopy (SEM) were made at $15 \mathrm{kV}$ with a Hitachi S-4800. The sample on the silicon substrate was covered with $5 \mathrm{~nm}$ of gold to enhance the conductivity and avoid sample charging under the electron beam. Magnetic measurements were carried out in a homemade Magneto-Optical Kerr Effect magnetometer with longitudinal geometry, equipped with an electromagnet of $0.2 \mathrm{~T}$ of maximum field. The measurement conditions were $4.1 \mathrm{~Hz}$ of frequency, a range up to $240 \mathrm{mT}$ and 200 averages. Each sample on silicon was oriented at different angles with respect to the applied magnetic field $(\mathrm{H})$, and the hysteresis loops were obtained by carefully positioning the laser spot $(10 \mu \mathrm{m})$ in the same point after each rotation. A Philips EM208S was used for the TEM measurements. The sample preparation was carried out by the same procedure, by depositing a droplet onto a thin amorphous carbon film on a TEM copper grid. The width of the chains and the particle size distribution was analyzed using standard software for digital electron microscope image processing, ImageJ. ${ }^{32}$ Off-axis Electron Holography was performed in an FEI TITAN Cube operated at $300 \mathrm{kV}$ and fitted with an S-Twin objective lens, an image Cs corrector (CETCOR from CEOS), and a motorized electrostatic biprism.

SEM images reveal that the assembly of magnetosomes deposited by drop coating on silicon substrates, remained well oriented in the form of elongated lines in the direction of the deposition-magnetic field $\left(\mathrm{H}_{\mathrm{axis}}\right)$ (see Figure $\mathrm{S} 4$ in supplementary material $\left.{ }^{30}\right)$. The distribution of chain-like objects over the silicon surface was relatively homogeneous with irregular spaces between the wide chains and small branches connecting
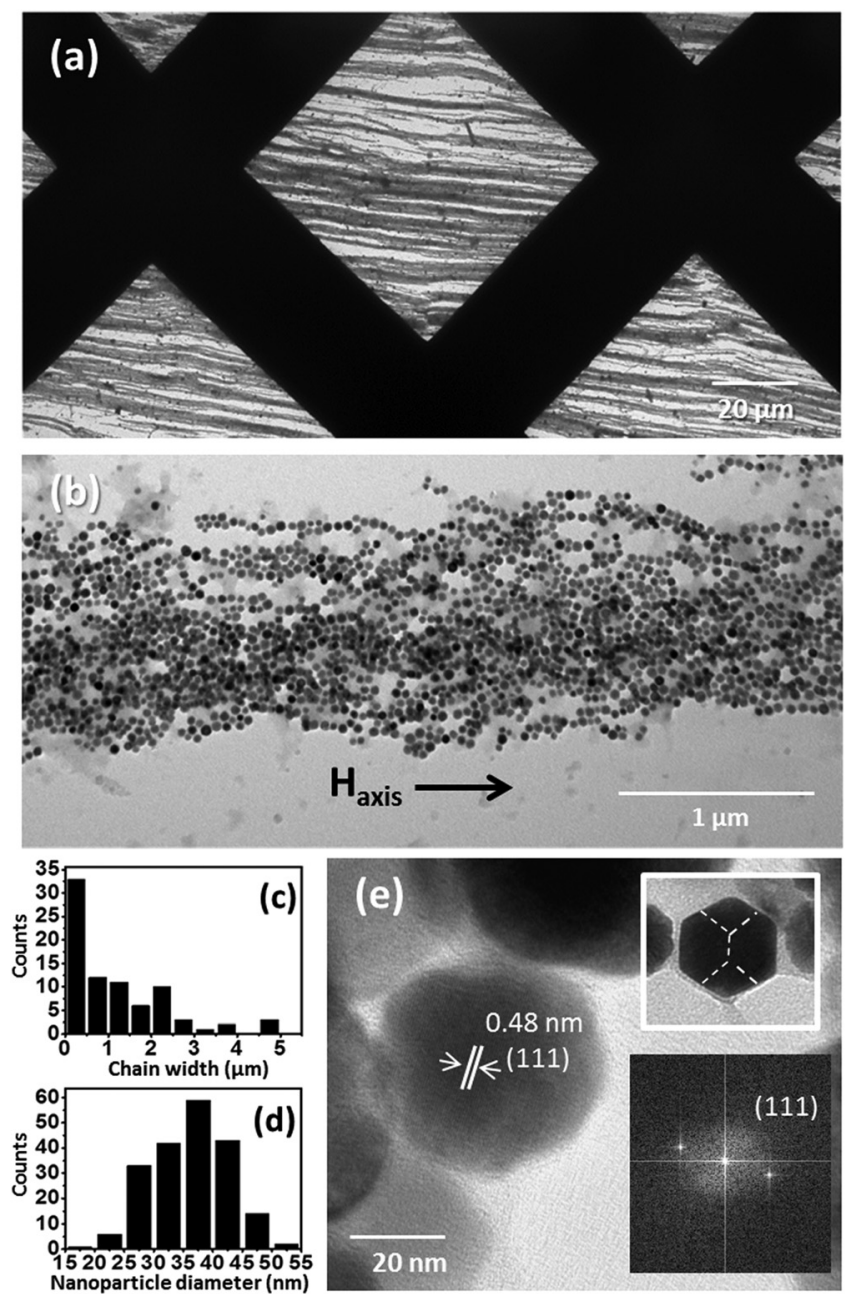

FIG. 1. TEM images of magnetosomes assembled on continuous carbon surface under an applied magnetic field of $0.4 \mathrm{~T}$ and dried under infrared light. (a) The image at low magnification show the distribution of magnetosomes chains on a film of continuous carbon, where it can be seen the squares delimited by the copper grid. (b) A detail of an assembly of magnetosomes. (c) Histogram of the chains width. (d) Histogram of the nanoparticle diameters and (e) HRTEM image of one magnetosome and the FFT of the image; the spots are related with $\{111\}$ planes. Inset: an enlarged view of one magnetosome where the truncated cubo-octahedral morphology is clearly observed; the lines are guide for the eyes. 
(a)
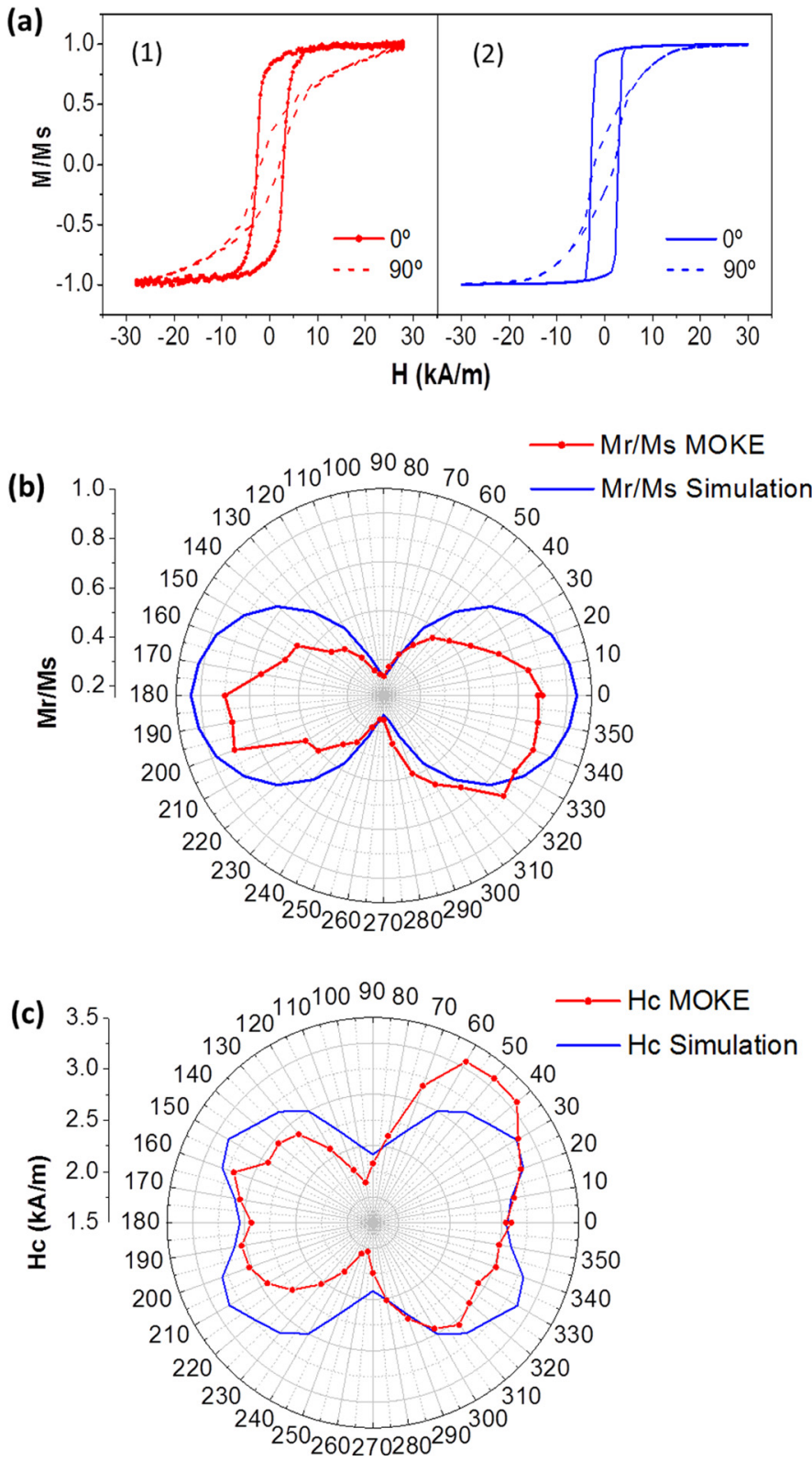

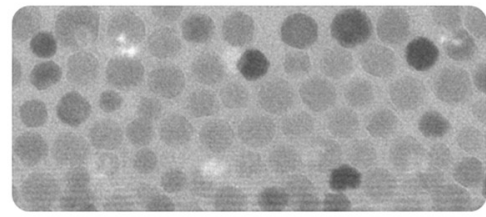

TOP VIEW OF ASSEMBLIES

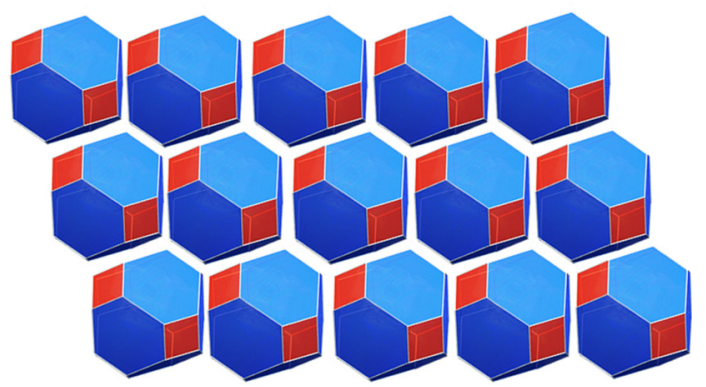

SIDE VIEW OF ASSEMBLIES

H
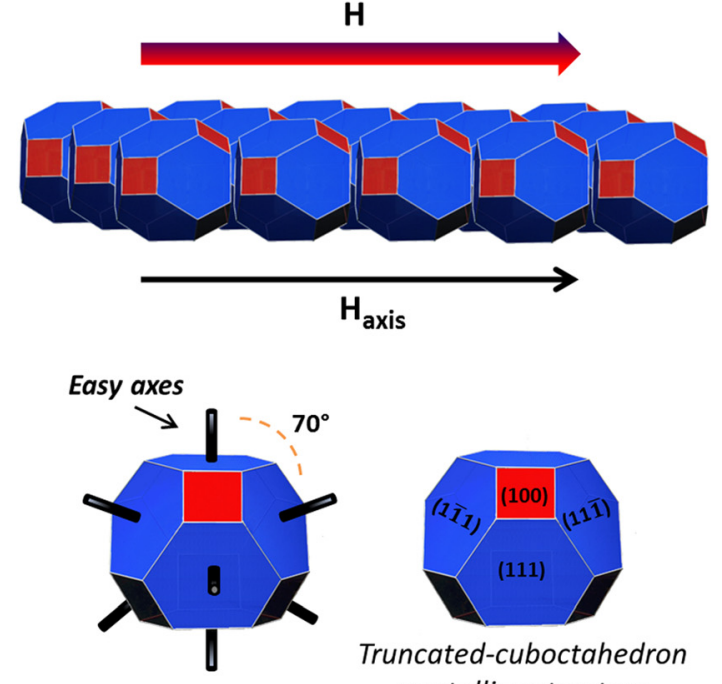

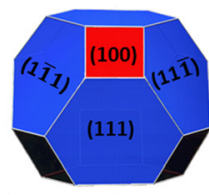

Truncated-cuboctahedron crystalline structure

FIG. 2. (a1) Experimental MOKE and (a2) theoretical hysteresis loops parallel, $0^{\circ}$, and perpendicular, $90^{\circ}$, to the axis of the magnetosomes chains; polar plot of the angular dependence of (b) the remanence, $\mathrm{Mr} / \mathrm{Ms}$, and (c) the coercivity, Hc. Right: Schematic representation of an ideal wide chain of assembled magnetosomes, considering the morphology of truncated-cuboctahedron. With arrows are drawn the directions of the applied magnetic field, H, during the MOKE measurements in parallel configuration and the deposition-magnetic field $\mathrm{H}_{\mathrm{axis}}$.

them. On the other hand, TEM measurements allow observing the assembly of magnetosomes on carbon surface (Figure 1). The chains are arranged on the carbon surface in a very homogeneous way, as can be seen in Figure 1(a): they form long lines in the direction of the $\mathrm{H}_{\mathrm{axis}}$ and cover a large area of the substrate, independent on the substrate used (more details in supplementary material ${ }^{30}$ ). This particular self-arrangement seems to be very reproducible as long as the deposition surface is sufficiently smooth and the same concentration and deposition-magnetic field are used (see Figure S4 of supplementary material ${ }^{30}$ ). The chain-shaped structures must be mainly attributed to the influence of deposition-magnetic field, which promotes the alignment of the magnetic dipoles to reduce the magnetostatic energy (similarly to previous reported works). ${ }^{33}$ Figure 1(b) shows in more detail the structure of a wide chain. Magnetosomes membranes prevent the particles from agglomerating or collapsing, acting as stabilizers and favoring a 2D arrangement. The thickness of lipid membrane is $\approx 2-4 \mathrm{~nm},{ }^{14}$ so that the minimum distance between magnetosomes was $\approx 6 \mathrm{~nm}$. The wide bands or chains showed widths ranging from $135 \mathrm{~nm}$ to $5000 \mathrm{~nm}$ (Figure 1(c)), with an average of around $500 \mathrm{~nm}$, and few single chains between them. The distribution of nanoparticle sizes can be seen in Figure 1(d) as a histogram, and the mean diameter is about $36 \pm 6 \mathrm{~nm}$. In addition, Figure 1(e) shows the high-resolution image (HRTEM) of one magnetosome. In the image, it is observed an interplanar distance of $0.48 \mathrm{~nm}$ that corresponds to the distance between $\{111\}$ planes of cubic magnetite, ${ }^{34}$ and the Fast Fourier Transformed (FFT) of the image whose spots represent the $\{111\}$ planes. The magnetosomes $($ size $\approx 36 \mathrm{~nm}$ ) show a truncated-cuboctahedron morphology (Figure 1(e) inset), formed by a combination of eight hexagonal $\{111\}$ and six square $\{100\}$ faces. This morphology, which is in agreement with previous studies about magnetosomes extracted from 
Magnetospirillum gryphiswaldense, ${ }^{35-37}$ favors a two-fold shape magnetic anisotropy with easy axes corresponding to [111] directions. ${ }^{12,36,38}$

MOKE in longitudinal geometry ${ }^{39}$ has been used to study the magnetization process of these structures as a function of the angle between chains and external magnetic field applied in the plane of the samples. Figure 2(a) shows the hysteresis loops obtained with the magnetic field applied parallel, $0^{\circ}$, and perpendicular, $90^{\circ}$, to the magnetosomes chain's axis, respectively. As expected, the magnetic response is strongly anisotropic but does not correspond to a pure uniaxial system: in the perpendicular orientation, the coercive field and the remanence are smaller than those of the parallel case, but they are far from being zero, as it should be in the uniaxial case with the easy axis oriented along the chains. The intensity of the resultant anisotropy can be quantified by the ratio of the parallel to the perpendicular reduced remanence $\gamma=\left(M_{r} / M_{s}\right)^{\perp} /\left(M_{r} / M_{s}\right)^{\|}$, being 0.31 instead of 0 . The existence of strong anisotropy in the two-dimensional arrangements is also evident from the polar representation of the reduced remanence (Figure 2(b)) and the coercitivity (Figure 2(c)). Both figures show a clear narrowing or waist corresponding to two equivalent minima located roughly at $90^{\circ}$ and $270^{\circ}$. The onset of two minima separated by $180^{\circ}$ marks a well-defined hard magnetic axis perpendicular to the chains in agreement with previous discussion.

In principle, these results can be explained either by a strong misalignment of uniaxial particles or as due to the existence of off-chains magnetic axes. The polar plot of the coercitivity, in Figure 2(c), presents some traces of a butterfly-like shape or a two-fold in-plane anisotropy, though in the present case, the two local minima at around $0^{\circ}$ and $180^{\circ}$ are much weaker or smoothed, than the others. This is characteristic of cubic or multiaxial anisotropy, so suggesting that the offchain easy axes are mostly pointing in some particular directions in the $2 \mathrm{D}$ arrangement. The explanation of these features seems to rely on the properties of the individual magnetite particles and on those of the self-assembling. On the one hand, the truncated cubo-octahedral shape is expected to imprint a cubic character to the anisotropy of individual particles, leading to 4 easy axes directed along the [111] directions, which is superimposed to the magnetocrystalline contribution that is expected to be smaller in any case. ${ }^{25,40} \mathrm{On}$ the other hand, nearest neighbors in the close-packed structure tend to orient themselves by opposing the hexagonal faces, leading to stronger dipolar interactions in those directions. ${ }^{41}$ It means that even in an ideal situation, assuming that each particle has an easy axis toward the deposition-magnetic field, $\mathrm{H}_{\text {axis }}$, the assembly should have local magnetic energy minima with certain tendency to lie at $70^{\circ}$ off the deposition field at several azimuthal angles, as sketched in Figure 2. As a consequence, the net energy landscape is expected to have several off-axes local minima that lead to non-zero remanence and coercive field in the in-plane perpendicular direction.

This hypothesis has been tested by performing simulations of the hysteresis loops. We have made use of a dynamical model where the energy landscape corresponds to a biaxial system, with one axis fixed along the deposition field and the second one representing the local "fluctuation" of the anisotropy caused by the individual anisotropy and/or dipolar interaction with neighbors. ${ }^{42}$ According to previous discussion, the angle
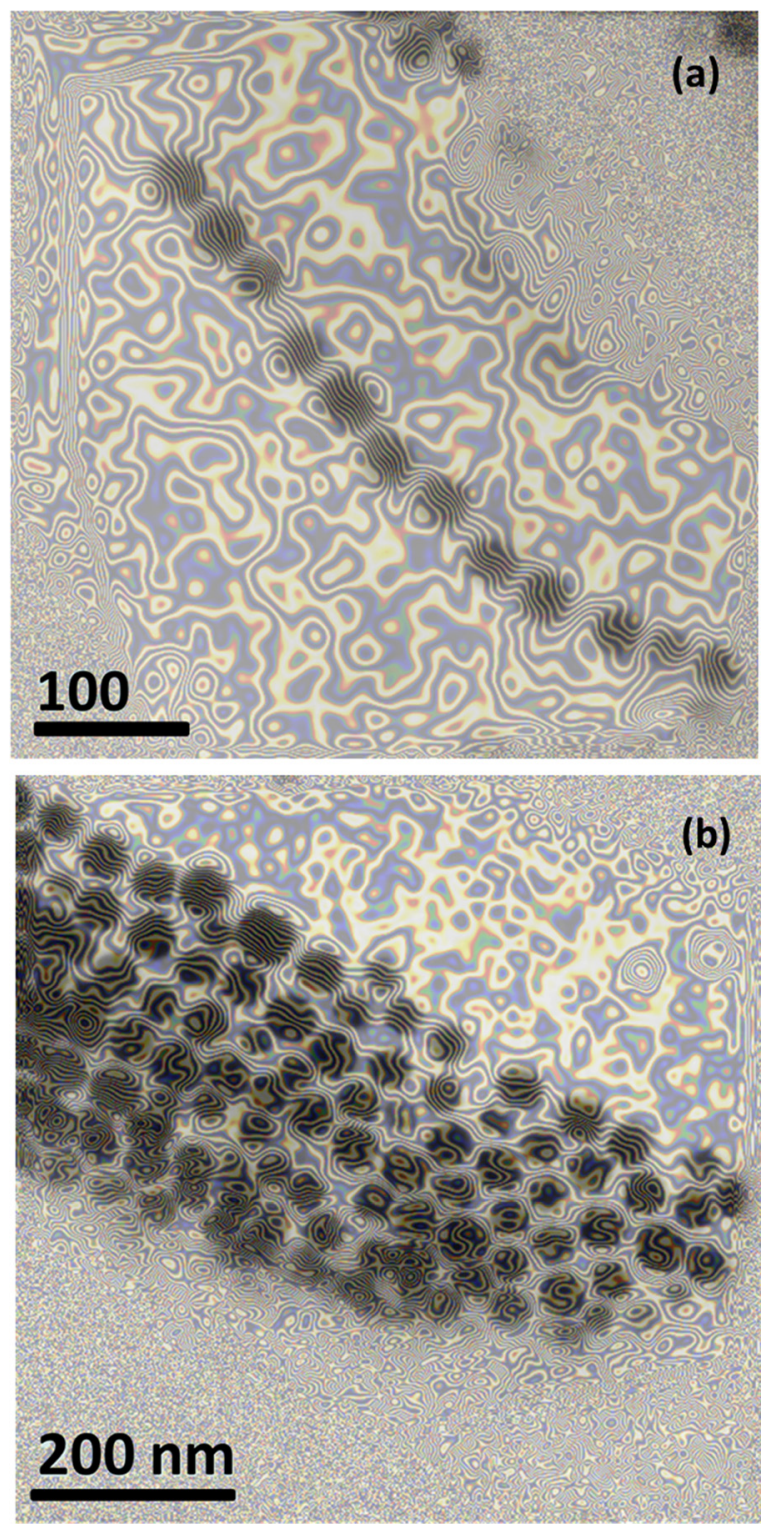

FIG. 3. Magnetic induced maps recorded using off-axis electron holography, showing remanent magnetic states at room temperature. (a) Single row of a linearly assembled magnetosomes. (b) Wide chains of assembled magnetosomes. The magnetic line contours are overlaid onto the TEM image of the magnetosomes chains.

between them was fixed to $70^{\circ}$, and the second axis was allowed to explore the whole azimuthal range. Details of the model are in the supplementary material, and the results are compared with the experimental data in Figures 2(a)-2(c). In these, the best agreement between experiments and modeling is achieved with uniaxial constants equal to $\mathrm{K}_{1}=3.8 \mathrm{~kJ} / \mathrm{m}^{3}$ and $\mathrm{K}_{2}=2.6 \mathrm{~kJ} / \mathrm{m}^{3}$, which are in the order of others values found in the literature for magnetosomes bound in chains. ${ }^{12}$ The estimated anisotropy energy ( $K v, v$ particle volume) corresponds with the dipolar interaction energy between two dipoles separated $\approx 60 \mathrm{~nm}$ (given by $\sim \frac{\mu_{0}}{4 \pi} \frac{2 \mu^{2}}{a^{3}}$ ). As observed in Figure 2(a), simulated loops match rather well with the experimental ones, predicting a nonzero hysteresis at the hard axis (at $90^{\circ}$ and $270^{\circ}$ ) and the existence of asymmetric minima in the polar plot of the coercivity, which are weaker along the deposition magnetic field or chain's main axis $\left(0^{\circ}\right.$ and $\left.180^{\circ}\right)$. Moreover, the model predicts a more simple 1-fold polar dependence of the remanence, which is in good accord with experimental data (Figure 2(b)). 
The off-axis electron holography maps (Figure 3) show the magnetic remanent state at room temperature of magnetosomes chains. These maps demonstrate the existence of strong dipolar magnetic interactions that promote different configuration depending on the magnetosomes arrangement. Figure 3(a) presents the magnetic field contour lines corresponding to a single chain of linearly assembled magnetosomes. These contour lines pass through the chain from one end to the other, indicating that each particle behaves as a single magnetic domain almost perfectly aligned with the neighbors. This demonstrates the existence of a well-defined easy axis parallel to the applied deposition-magnetic field, originated by dipolar interactions. ${ }^{43,44}$ In general, it was observed that single chains are formed by less than twelve magnetosomes, which behave as a single dipole. ${ }^{45}$ However, the existence of small particles or even defects seems to break the linear arrangement (see Figure S6 in supplementary material ${ }^{30}$ ). Figure 3(b) shows the map of the magnetic induction field inside a wide chain of magnetosomes, composed of around 7-8 rows. It is to highlight that the magnetic field lines tend to lose the longitudinal pattern inside the chain-like cluster, leading to an intricate network of twisted lines with a large net transversal magnetic moment. This is in agreement with previous discussion because it is compatible with the existence of local off-axis energy minima. The density of the contour lines indicates that the magnetic dipolar interaction becomes weaker inside of the wide chains so that particles easily decouple from the neighbors and the system tends to lose a well-defined longitudinal anisotropy axis. This behavior is in contrast with some reported works on strongly uniaxial magnetic nanoparticles, ${ }^{9}$ where a perfect alignment of dipoles was observed.

In summary, we have been able to prepared selfassembled biomineralized-magnetite nanoparticles. The so obtained magnetic arrangement has an easy axis oriented in the direction of the deposition field, but it has been shown to retain some magnetic properties of the magnetite single crystals, favoured by the close-packing properties of cubooctahedral shaped particles. This fact mainly affects the 2D arrangements, where the magnetic induction lines have been shown to possess strong off-axis orientation. This misalignment seems to be favoured by the proliferation of local energy minima, characteristic of single domains of cubic symmetry. The magnetic anisotropy is mainly determined by the short range order of the $2 \mathrm{D}$ arrangements and has been well reproduced by a single particle approach.

A. M. Huízar-Félix wishes to thank CONACYT for her postdoctoral fellowship. This work has been partially supported by the Project MAT2014-55049-C2-1-R from the Spanish Government.

${ }^{1}$ G. M. Whitesides and B. Grzybowski, Science 295, 2418 (2002).

${ }^{2}$ K. Thorkelsson, P. Bai, and T. Xu, Nano Today 10, 48 (2015).

${ }^{3}$ M. Boncheva, D. A. Bruzewicz, and G. M. Whitesides, Pure Appl. Chem. 75, 621 (2003).

${ }^{4}$ A. Wei, T. Kasama, and R. E. Dunin-Borkowski, J. Mater. Chem. 21, 16686 (2011)

${ }^{5}$ W. C. Ruder, C. P. Hsu, B. D. Edelman, R. Schwartz, and P. R. LeDuc, Appl. Phys. Lett. 101, 063701 (2012).

${ }^{6}$ R. Hergt and S. Dutz, J. Magn. Magn. Mater. 311, 187 (2007).
${ }^{7}$ Y. Men, W. Wang, P. Xiao, J. Gu, A. Sun, Y. Huang, J. Zhang, and T. Chen, RSC Adv. 5, 31519 (2015).

${ }^{8}$ M. Varón, L. Peña, L. Balcells, V. Skumryev, B. Martinez, and V. Puntes, Langmuir 26, 109 (2010)

${ }^{9}$ M. Varón, M. Beleggia, T. Kasama, R. J. Harrison, R. E. DuninBorkowski, V. F. Puntes, and C. Frandsen, Sci. Rep. 3, 1234 (2013).

${ }^{10}$ S. A. Majetich, T. Wen, and R. A. Booth, ACS Nano 5, 6081 (2011).

${ }^{11}$ J. Alonso, M. L. Fdez-Gubieda, J. M. Barandiarán, A. Svalov, L. Fernández Barquín, D. Alba, and I. Orue, Phys. Rev. B 82, 054406 (2010).

${ }^{12}$ E. Alphandéry, Y. Ding, A. T. Ngo, Z. L. Wang, L. F. Wu, and M. P. Pileni, ACS Nano 3, 1539 (2009).

${ }^{13}$ D. A. Bazylinski, ASM News 61, 337 (1995).

${ }^{14}$ D. A. Bazylinski and R. B. Frankel, Nat. Rev. Microbiol. 2, 217 (2004).

${ }^{15}$ H. Gojzewski, M. Makowski, A. Hashim, P. Kopcansky, Z. Tomori, and M. Timko, Scanning 34, 159 (2012).

${ }^{16}$ E. Alphandery, S. Faure, O. Seksek, F. Guyot, and I. Chebbi, ACS Nano 5, 6279 (2011).

${ }^{17}$ S. Mériaux, M. Boucher, B. Marty, Y. Lalatonne, S. Prévéral, L. Motte, C. T. Lefèvre, F. Geffroy, F. Lethimonnier, M. Péan, D. Garcia, G. AdryanczykPerrier, D. Pignol, and N. Ginet, Adv. Healthcare Mater. 4, 1076 (2015).

${ }^{18}$ B. M. Moskowitz, R. B. Frankel, and D. A. Bazylinski, Earth Planet. Sci. Lett. 120, 283 (1993).

${ }^{19}$ B. M. Moskowitz, R. B. Frankel, D. A. Bazylinski, H. W. Jannasch, and D. R. Lovley, Geophys. Res. Lett. 16, 665, doi:10.1029/ GL016i007p00665 (1989).

${ }^{20}$ C. Carvallo, S. Hickey, D. Faivre, and N. Menguy, Earth, Planets Space 61, 143 (2009).

${ }^{21}$ R. E. Kopp, C. Z. Nash, A. Kobayashi, B. P. Weiss, D. A. Bazylinski, and J. L. Kirschvink, J. Geophys. Res. 111, B12S25, doi:10.1029/2006JB004529 (2006).

${ }^{22}$ A. P. Philipse and D. Maas, Langmuir 18, 9977 (2002).

${ }^{23}$ C. Carvallo, P. Sainctavit, M. A. Arrio, N. Menguy, Y. Wang, G. OnaNguema, and S. Brice-Profeta, Am. Mineral. 93, 880 (2008).

${ }^{24}$ M. Charilaou, J. Kind, I. García-Rubio, D. Schüler, and A. U. Gehring, Appl. Phys. Lett. 104, 112406 (2014).

${ }^{25}$ M. Charilaou, K. K. Sahu, D. Faivre, A. Fischer, I. García-Rubio, and A. U. Gehring, Appl. Phys. Lett. 99, 182504 (2011).

${ }^{26}$ A. Körnig, M. A. Hartmann, C. Teichert, P. Fratzl, and D. Faivre, J. Phys. D: Appl. Phys. 47, 235403 (2014).

${ }^{27}$ M. L. Fdez-Gubieda, A. Muela, J. Alonso, A. Garcia-Prieto, L. Olivi, R. Fernandez-Pacheco, and J. M. Barandiarán, ACS Nano 7, 3297 (2013).

${ }^{28}$ D. Schüler, R. Uhl, and E. A. Baeuerlein, FEMS Microbiol. Lett. 132, 139 (1995).

${ }^{29}$ U. Heyen and D. Schüler, Appl. Microbiol. Biotechnol. 61, 536 (2003).

${ }^{30}$ See supplementary material at http://dx.doi.org/10.1063/1.4941835 for experimental details, study of optimal parameters for the deposition and calculation of the hysteresis loops.

${ }^{31}$ K. Grünberg, C. Wawer, B. M. Tebo, and D. Schuler, Appl. Environ. Microbiol. 67, 4573 (2001).

${ }^{32}$ C. A. Schneider, W. S. Rasband, and K. W. Eliceiri, Nat. Methods 9, 671 (2012).

${ }^{33}$ S. A. Majetich and M. Sachan, J. Phys. D: Appl. Phys. 39, R407 (2006).

${ }^{34}$ Joint Committee on Powder Diffraction Standards (JCPDS), File No. 19-0629.

${ }^{35}$ D. A. Bazylinski, A. J. Garratt-Reed, and R. B. Frankel, Microsc. Res. Tech. 27, 389 (1994).

${ }^{36}$ S. Mann, R. B. Frankel, and R. P. Blakemore, Nature 310, 405 (1984).

${ }^{37}$ E. Katzmann, M. Eibauer, W. Lin, Y. Pan, J. M. Plitzko, and D. Schüler, Appl. Environ. Microbiol. 79, 7755 (2013).

${ }^{38}$ T. J. Meurig, E. T. Simpson, T. Kasama, and R. E. Dunin-Borkowski, Acc. Chem. Res. 41, 665 (2008).

${ }^{39}$ D. A. Allwood, G. Xiong, M. D. Cooke, and R. P. Cowburn, J. Phys. D: Appl. Phys. 36, 2175 (2003).

${ }^{40}$ R. E. Dunin, M. R. McCartney, R. B. Frankel, D. A. Bazylinski, M. Pósfai, and P. R. Buseck, Science 282, 1868 (1998).

${ }^{41}$ N. A. Usov, M. L. Fdez-Gubieda, and J. M. Barandiarán, J. Appl. Phys. 113, 023907 (2013).

${ }^{42}$ N. A. Usov and J. M. Barandiarán, J. Appl. Phys. 112, 053915 (2012).

${ }^{43}$ R. E. Dunin-Borkowski, T. Kasama, A. Wei, S. L. Tripp, M. J. Hÿtch, E. Snoeck, R. J. Harrison, and A. Putnis, Microsc. Res. Tech. 64, 390 (2004).

${ }^{44}$ R. E. Dunnin-Borkowski, M. R. McCartney, M. Pósfai, R. B. Frankel, D. A. Bazylinski, and P. R. Buseck, Eur. J. Mineral. 13, 671 (2001).

${ }^{45}$ M. Bennet, L. Bertinetti, R. K. Neely, A. Schertel, A. Körnig, C. Flors, F. D. Müller, D. Schüler, S. Klumpp, and D. Faivre, Faraday Discuss. 181, 71 (2015). 